\title{
Biomechanical Analysis of Biodegradable Cervical Plates Developed for Anterior Cervical Discectomy and Fusion
}

\author{
Pyung Goo Cho ${ }^{1}$, Gyu Yeul ji ${ }^{2}$, Sang Hyuk Park ${ }^{3}$, Dong Ah Shin ${ }^{2}$ \\ ${ }^{1}$ Department of Neurosurgery, Bundang Jesaeng Hospital, Seongnam, Korea \\ ${ }^{2}$ Department of Neurosurgery, Yonsei University College of Medicine, Seoul, Korea \\ ${ }^{3}$ Department of Neurosurgery, Yonsei Barun Hospital, Seoul, Korea
}

Study Design: In-vitro biomechanical investigation.

Purpose: To evaluate the biomechanical effects of the degeneration of the biodegradable cervical plates developed for anterior cervical discectomy and fusion (ACDF) on fusion and adjacent levels.

Overview of Literature: Biodegradable implants have been recently introduced for cervical spine surgery. However, their effectiveness and safety remains unclear.

Methods: A linear three-dimensional finite element (FE) model of the lower cervical spine, comprising the C4-C6 vertebrae was developed using computed tomography images of a 46-year-old woman. The model was validated by comparison with previous reports. Four models of ACDF were analyzed and compared: (1) a titanium plate and bone block (Tita), (2) strong biodegradable plate and bone block (PLA-4G) that represents the early state of the biodegradable plate with full strength, (3) weak biodegradable plate and bone block (PLA-1G) that represents the late state of the biodegradable plate with decreased strength, and (4) stand-alone bone block (Bloc). FE analysis was performed to investigate the relative motion and intervertebral disc stress at the surgical (C5-C6 segment) and adjacent (C4-C5 segment) levels.

Results: The Tita and PLA-4G models were superior to the other models in terms of higher segment stiffness, smaller relative motion, and lower bone stress at the surgical level. However, the maximal von Mises stress at the intervertebral disc at the adjacent level was significantly higher in the Tita and PLA-4G models than in the other models. The relative motion at the adjacent level was significantly lower in the PLA-1G and Bloc models than in the other models.

Conclusions: The use of biodegradable plates will enhance spinal fusion in the initial stronger period and prevent adjacent segment degeneration in the later, weaker period.

Keywords: Absorbable implants; Cervical spine; Spinal fusion; Finite element analysis; Intervertebral disc degeneration

\section{Introduction}

Anterior cervical discectomy and fusion (ACDF) is a safe and effective therapy for degenerative or traumatic conditions affecting the cervical spine [1]. Several methods have been used to perform ACDF; the method that involves the

Received Jan 25, 2018; Revised Mar 4, 2018; Accepted Apr 17, 2018

Corresponding author: Dong Ah Shin

Department of Neurosurgery, Yonsei University College of Medicine, 50-1 Yonsei-ro, Seodaemun-gu, Seoul 03722, Korea

Tel: +82-2-2228-2150, Fax: +82-2-393-9979, E-mail: cistern@yuhs.ac 
addition of an anterior plate is currently the most commonly used. Additional anterior plate fixation reportedly reduces the cage subsidence rate and enhances the fusion rate following ACDF [2-4]. However, complications related to ACDF, such as dysphagia, acceleration of adjacent segment degeneration (ASD), and postoperative image artifacts of computed tomography (CT) and magnetic resonance imaging (MRI), are common and reportedly occur in up to $14 \%$ of all cases [5-7]. Patients may develop dysphagia following ACDF at any stage postoperatively [8-11]. In addition, titanium plates are commonly used for ACDF; this may result in imaging artifacts due to the resultant paramagnetic effect [12]. Moreover, ASD is the main complication that warrants consideration after ACDF using the anterior plate system [6].

Recently, biodegradable plate and screw systems have been developed to prevent these complications $[13,14]$. The introduction of these biodegradable systems may provide a novel benefit for reducing some long-term complications and removing imaging artifacts associated with the use of titanium-based systems. However, the effectiveness and safety of these biodegradable systems remains unclear. The present study aimed to evaluate the biomechanical differences between a biodegradable and titanium cervical plate in the operated and adjacent cervical spine after ACDF.

\section{Materials and Methods}

\section{Finite element model}

A three-dimensional (3D) non-linear finite element (FE) model of the cervical spine from the $\mathrm{C} 4$ to $\mathrm{C} 6$ vertebrae was created. To ensure model geometry, a high-resolution CT scan (1.0-mm thickness) was performed for a 46-yearold woman, height $160 \mathrm{~cm}$, with neck pain. This study was approved by the Institutional Review Board in medical center of Yonsei University as "exempt from consideration" (IRB no., 2017-2466-01). The patient received detailed study information and provided informed consent for this scan. We imported the digital imaging and communications in medicine data into Mimics (Materialise, Leuven, Belgium) and generated a 3D model. Then, the spine model was imported into IA-FEMesh (The University of Iowa, Iowa City, IA, USA), and an FE mesh was generated for analysis. ABAQUS (Dassault System, Paris, France) was used to simulate the surgical scenarios with the spine model. The FE model comprised three cervi-

Table 1. Material properties of the finite element model of the cervical spine

\begin{tabular}{|c|c|c|c|}
\hline Variable & Young's modulus $\left(\mathrm{N} / \mathrm{mm}^{2}\right)$ & Poisson's coefficient & Cross-section $\left(\mathrm{mm}^{2}\right)$ \\
\hline Cortical bone & 12,000 & 0.3 & \\
\hline Cancellous bone & 100 & 0.2 & \\
\hline Posterior arch & 6,000 & 0.3 & \\
\hline Endplate & 300 & 0.3 & \\
\hline Annulus fibrosus & 2.5 & 0.45 & \\
\hline Nucleus pulposus & 1 & 0.499 & \\
\hline Anterior longitudinal ligament & 10 & & 0.5 \\
\hline Posterior longitudinal ligament & 20 & & 0.5 \\
\hline Capsular ligament & 20 & & 0.6 \\
\hline Ligamentum flavum & 50 & & 0.4 \\
\hline Interspinous ligament & 3 & & 3 \\
\hline Supraspinous ligament & 3 & & 5 \\
\hline Tita & 10,000 & 0.3 & \\
\hline PLA-4G & 4,000 & 0.3 & \\
\hline PLA-1G & 1,000 & 0.3 & \\
\hline Bloc & 10,000 & 0.3 & \\
\hline
\end{tabular}

Tita, a titanium plate and bone block; PLA-4G, strong biodegradable plate and bone block; PLA-1G, weak biodegradable plate and bone block; Bloc, stand-alone bone block. 
cal vertebrae, two intervertebral discs, two endplates for each level, and six spinal ligaments (Fig. 1). The spinal ligaments included the anterior longitudinal ligament, posterior longitudinal ligament, ligamentum flavum, capsular ligament, interspinous ligament, and supraspinous ligament. The vertebral bodies comprised the outer cortical bone with shell elements (1-mm thickness) and inner trabecular bone with hexahedral elements. The posterior column was modeled as one part. The facet contact surface and endplates were composed of hexahedral elements (1-mm thickness). The intervertebral disc was composed of a nucleus pulposus and an annulus fibrosus. The annulus fibrosus was designed to include four layers. The spinal ligaments were modeled using truss elements. Surface-tosurface contact was used to simulate the interaction between the articulating surfaces of the facet joints. The entire vertebral body, endplate, and intervertebral disc were assembled with tie constraints at the interfacing surfaces. The properties of all elements were assigned, as shown in Table 1 . We used the parameters suggested in a previous study $[15,16]$. Linear elasticity was applied to the bone, intervertebral disc, and cartilaginous structures. The spinal ligaments were designed as bilinear with a specific yield strain and stress values.

\section{Finite element model of anterior cervical discectomy and fusion}

To compare the biomechanical changes after ACDF, four types of surgical models were developed based on the validated intact spine model: (1) ACDF using a titanium plate and bone block (Tita) (Fig. 1A), (2) ACDF using a strong biodegradable plate and bone block that represents the early state of the biodegradable plate with full strength (PLA-4G) (Fig. 1B), (3) ACDF using a weak biodegradable plate and bone block that represents the late state of the biodegradable plate with decreased strength (PLA-1G) (Fig. 1C), and (4) ACDF using a stand-alone bone block (Bloc) (Fig. 1). Both plate and screw systems had the same dimension. The length, width, and thickness of the plates were 22,16 , and $2 \mathrm{~mm}$, respectively. The length and diameter of the screws were 16 and $3.5 \mathrm{~mm}$, respectively. The depth, width, and height of the bone blocks were 7,12 , and $6 \mathrm{~mm}$, respectively. Screws were entirely tied to the vertebral bodies. A tie constraint was also applied to the bone block and vertebral bodies to simulate complete fusion.

\section{Boundary and loading conditions}

The lower facet surface of the C6 vertebra was completely fixed in all directions. A reference point was created and constrained to the surface nodes of the $\mathrm{C} 4$ vertebra. Pure unconstrained 2-Nm flexion, 2-Nm extension, 2-Nm lateral bending, and $2-\mathrm{Nm}$ torsion moments under $6-\mathrm{N}$ axial compression were applied to the reference point in a stepwise manner. To reach the target moment, 10 load steps were applied. The range of motion (ROM) calculated at the endpoint of the loading cycle was compared with that calculated in previous human studies [15,17-19]. The $\mathrm{ROM}$ and intradiscal pressure were examined for all four motions generated in the fusion (C5-C6) and the adjacent levels (C4-C5). The four motions comprised flexion and extension in the sagittal plane, lateral bending in the
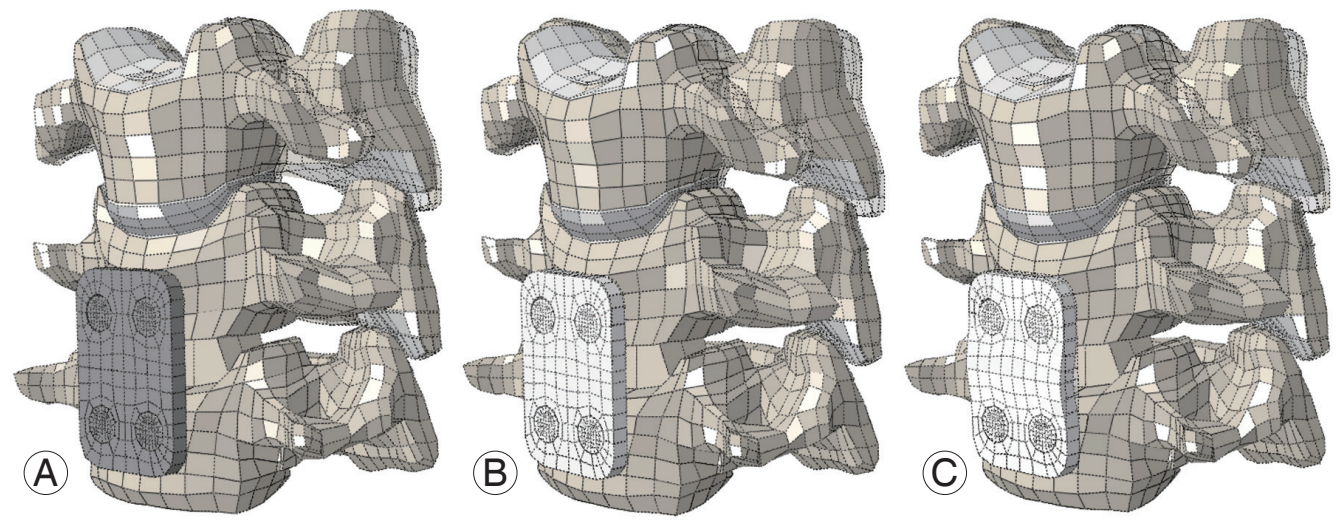

Fig. 1. Comparison of the three cervical plates in the 2-Nm flexion moment (isometric views). Spinal ligaments are hidden. (A) A titanium plate and bone block (Tita model); (B) a strong biodegradable plate and bone block (PLA-4G model); and (C) a weak biodegradable plate and bone block (PLA-1G model). 
Table 2. Model validation after comparison of the calculated range of motion values of the finite element models from previous studies

\begin{tabular}{lrccc} 
Variable & Current model & Maurel et al. [20] & Moroney et al. [21] & Pelker et al. [19] \\
Flexion & $-7.7 \pm 1.1$ & $-7.0 \pm 1.3$ & $-5.6 \pm 1.8$ & $-6.1 \pm 3.9$ \\
Extension & $7.3 \pm 1.5$ & $7.5 \pm 1.2$ & $3.5 \pm 1.9$ & $3.5 \pm 1.8$ \\
Lateral bending & $4.9 \pm 0.0$ & $5.4 \pm 0.4$ & $4.7 \pm 3.0$ & $4.7 \pm 0.8$ \\
Torque & $4.2 \pm 1.1$ & $4.1 \pm 0.7$ & $1.9 \pm 0.7$ & $3.9 \pm 1.4$ \\
\hline
\end{tabular}

Table 3. The calculated range of motion values of the finite element models in flexion, extension, lateral bending, and torque motions at the operation level (C5-C6)

\begin{tabular}{|c|c|c|c|c|c|}
\hline Variable & Control & Tita & PLA-4G & PLA-1G & Bloc \\
\hline Flexion & $5.15^{\circ}$ & $0.09^{\circ}$ & $1.10^{\circ}$ & $1.67^{\circ}$ & $3.28^{\circ}$ \\
\hline Extension & $7.19^{\circ}$ & $0.08^{\circ}$ & $0.57^{\circ}$ & $0.57^{\circ}$ & $3.08^{\circ}$ \\
\hline Lateral bending & $2.65^{\circ}$ & $0.11^{\circ}$ & $0.36^{\circ}$ & $0.36^{\circ}$ & $0.58^{\circ}$ \\
\hline Torque & $5.24^{\circ}$ & $0.05^{\circ}$ & $0.07^{\circ}$ & $0.07^{\circ}$ & $0.08^{\circ}$ \\
\hline
\end{tabular}

Tita, a titanium plate and bone block; PLA-4G, strong biodegradable plate and bone block; PLA-1G, weak biodegradable plate and bone block; Bloc, stand-alone bone block.

coronal plane, and torsion in the axial plane. We also examined the maximal von Mises stress of the intervertebral disc and implant at the operative level (C5-C6) and adjacent intervertebral disc level (C4-C5).

\section{Results}

\section{Model validation}

The intact model was compared with the data from previous trials to assess model validity [19-21]. The calculated ROM values for the FE model were compared with those in previous studies (Table 2). Experimental and simulated loading protocols were identical to those in the study by Maurel et al. [20], and our data were within $10 \%$ of the mean values they reported (Table 2). Thus, we confirmed the validity of our FE model of the cervical spine.

\section{Changes in range of motion}

The ROM of all four motions decreased at the fusion level (C5-C6 level) in all the ACDF models (Table 3). Calculated ROM values of FE models in the control model were $5.15^{\circ}$ in flexion motion, $7.19^{\circ}$ in extension motion, $5.24^{\circ}$ in torsion motion, and $2.65^{\circ}$ in the lateral bending motion at the operative level (C5-C6). All four models showed limited neck motion after ACDF surgery. These

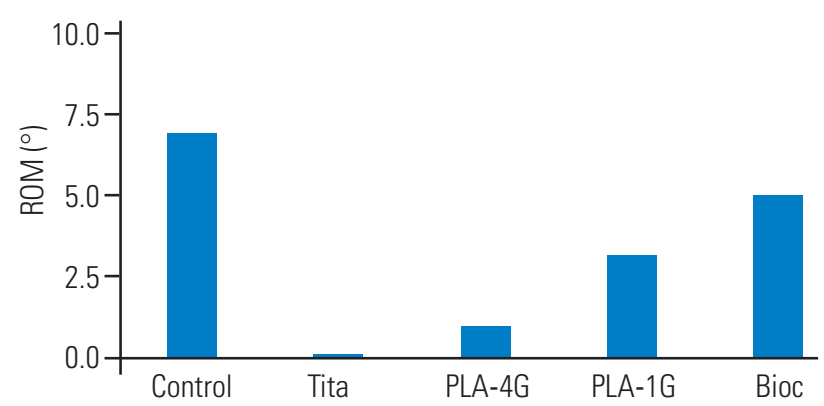

Fig. 2. ROM at the fusion level (C5-C6). The ROM is the difference in the angle between the flexion and the extension views. Tita, a titanium plate and bone block; PLA-4G, strong biodegradable plate and bone block; PLA-1G, weak biodegradable plate and bone block; Bloc, stand-alone bone block; ROM, range of motion.

data showed that the ROMs of flexion, extension, and lateral bending motions were further reduced after adding the plate system. The Tita and PLA- $4 \mathrm{G}$ models resulted in the greatest ROM limitations compared to those of the PLA-1G and Bloc models. The use of the PLA- $1 \mathrm{G}$ and Bloc models also exerted a reduction in the ROM, but to a lesser degree (Fig. 2).

ROM values at the adjacent level (C4-C5 level) were $5.99^{\circ}$ in flexion motion, $5.63^{\circ}$ in extension motion, $4.94^{\circ}$ in torsion, and $3.33^{\circ}$ in the lateral bending motion in the control model (Table 4). In all four implanted models, the ROMs of all directions at the adjacent level were mostly like that of the intact spine (Fig. 3). These data showed 
Table 4. The calculated range of motion values of the finite element models in flexion, extension, lateral bending, and torque motions at the adjacent level (C4-C5)

\begin{tabular}{lccccc} 
Variable & Control & Tita & PLA-4G & PLA-1G & Bloc \\
\hline Flexion & $5.99^{\circ}$ & $6.02^{\circ}$ & $6.02^{\circ}$ & $6.03^{\circ}$ & $6.06^{\circ}$ \\
Extension & $5.63^{\circ}$ & $5.52^{\circ}$ & $5.52^{\circ}$ & $5.52^{\circ}$ & $5.56^{\circ}$ \\
Lateral bending & $3.33^{\circ}$ & $3.51^{\circ}$ & $3.47^{\circ}$ & $3.47^{\circ}$ & $3.44^{\circ}$ \\
Torque & $4.94^{\circ}$ & $4.70^{\circ}$ & $4.70^{\circ}$ & $4.70^{\circ}$ & $5.21^{\circ}$ \\
\hline
\end{tabular}

Tita, a titanium plate and bone block; PLA-4G, strong biodegradable plate and bone block; PLA-1G, weak biodegradable plate and bone block; Bloc, stand-alone bone block.

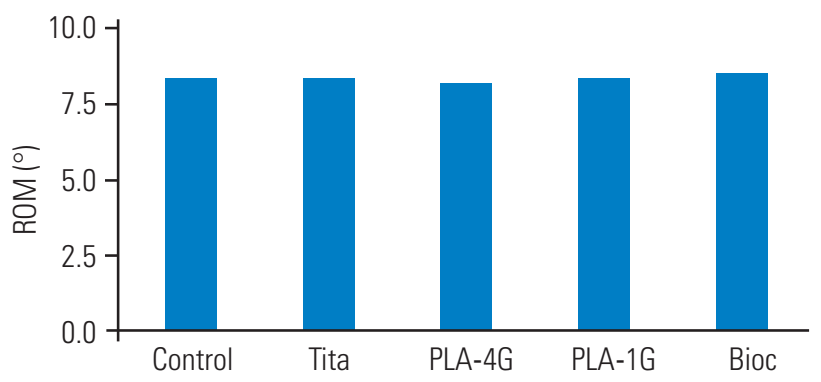

Fig. 3. ROM at the adjacent level (C4-C5). The ROM is the difference in the angle between the flexion and the extension views. Tita, a titanium plate and bone block; PLA-4G, strong biodegradable plate and bone block; PLA-1G, weak biodegradable plate and bone block; Bloc, stand-alone bone block; ROM, range of motion.

that the strength of the implant in the fusion level does not influence the ROM of the adjacent segment.

\section{Changes in the maximal von Mises stress in the fu- sion-level block bone}

The maximal von Mises stress values in the fusion-level block bone obtained with the Tita $\left(38.4 \mathrm{~N} / \mathrm{mm}^{2}\right)$ and PLA-4G $\left(40.4 \mathrm{~N} / \mathrm{mm}^{2}\right)$ models were similar. The PLA-1G and Bloc models had higher values: $47.1 \mathrm{~N} / \mathrm{mm}^{2}$ and 63.1 $\mathrm{N} / \mathrm{mm}^{2}$, respectively. The maximal von Mises stress in the block bone was the highest in the Bloc model. Excessive stress on the block bone may induce subsidence, destruction, and consequent non-union (Fig. 4).

\section{Changes in the maximal von Mises stress in the adja- cent intervertebral discs}

The maximal von Mises stress at the adjacent level was higher in the Tita $\left(5.03 \mathrm{~N} / \mathrm{mm}^{2}\right)$ and PLA-4G $(5.04 \mathrm{~N} /$ $\mathrm{mm}^{2}$ ) models than in the other models; however, it was lower in the PLA-1G $\left(4.92 \mathrm{~N} / \mathrm{mm}^{2}\right)$ and Bloc $(4.93 \mathrm{~N} /$

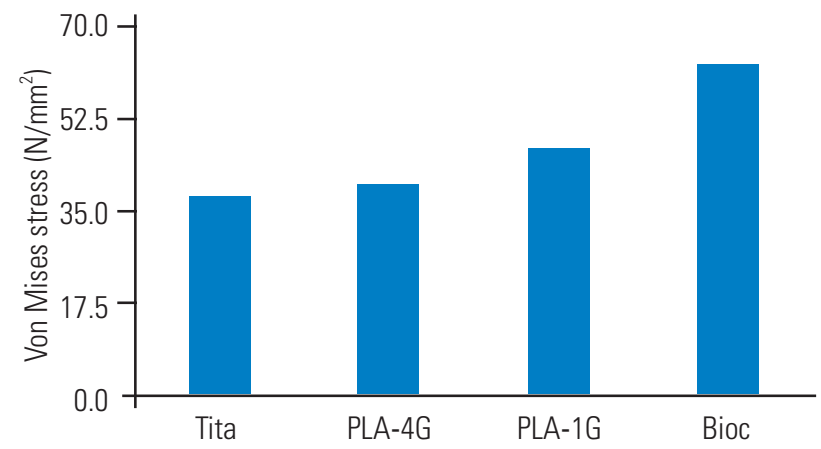

Fig. 4. The maximal von Mises stress in the bone block at the operative level (C5-C6). Tita, a titanium plate and bone block; PLA-4G, strong biodegradable plate and bone block; PLA-1G, weak biodegradable plate and bone block; Bloc, stand-alone bone block.

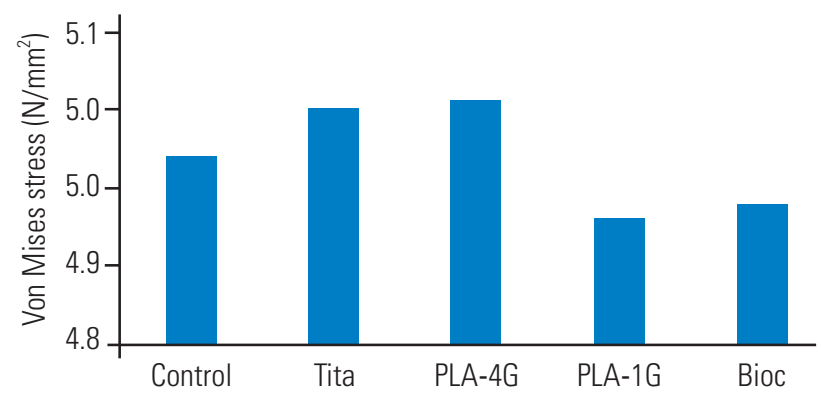

Fig. 5. The maximal von Mises stress at the adjacent intervertebral disc (C4-C5). Tita, a titanium plate and bone block; PLA-4G, strong biodegradable plate and bone block; PLA-1G, weak biodegradable plate and bone block; Bloc, stand-alone bone block.

$\mathrm{mm}^{2}$ ) models than in the other models. High stress in the adjacent intervertebral disc may contribute to ASD (Fig. 5).

\section{Discussion}

ACDF with anterior plate support has been widely used in cervical degenerative diseases. However, several complications have been reported after ACDF. Common complications include the development of isolated postoperative 
dysphagia, postoperative hematoma, ASD, symptomatic recurrent laryngeal nerve palsy, dural penetration, esophageal perforation, Horner syndrome, instrumentation breakage, and wound infection $[6,8,11,22,23]$. In addition, metallic instruments cause excessive image artifacts on postoperative MRI [24]. A biodegradable plate system has been introduced to overcome these problems. Biodegradable plate systems are typically absorbed within 6 months to 1 year postoperatively, and if dysphagia occurs, it does not persist after this period $[13,25,26]$. Dysphagia can be reduced because the mass effect of the plate disappears. In addition, it has no paramagnetic effect, making it advantageous in the evaluation of postoperative MRI scans. However, the effects of degradation of the biodegradable plate system on fusion and the adjacent levels have not yet been studied.

We assumed that the degradable plate would progress through three different stages. In the initial stage, the plate exhibits full stiffness without any degradation (PLA$4 \mathrm{G}$ model). In the second stage, partial degradation and structural weakness are observed (PLA-1G model). In the third stage, the system is fully degraded and disappears (Bloc model). This study evaluated the effect of biodegradable system on the fusion and adjacent levels at each stage by the FE model.

The cage-alone ACDF method is associated with some complications, including cage subsidence and a lower fusion rate, compared to ACDF using plate reinforcement [2]. This study showed that the von Mises stress on the intervertebral bone graft was higher in the Bloc model than that in all the other models (Fig. 4). The additional plate shares the loading on the interbody bone block. This suggests that the fusion rate in the plate system will be increased by preventing destruction of the bone block until the fusion of the interbody bone. This result is in agreement with previous findings that have shown that stand-alone bone fusion resulted in several complications, such as bone breakage, subsidence, and non-union [27]. However, the von Mises stress value shows that the stress applied to the adjacent level increases with the use of the plate system (Fig. 5). This indicates that while the fusion level reduces the stress on the bone block, the probability of ASD occurrence increases. ASD occurs more frequently when the plate system is used [28]. In the case of biodegradable plates, as shown in the initial PLA- $4 \mathrm{G}$ model, the stress value of the fusion level decreases to disperse the pressure until the bone block is fused. After the fusion, the stress value, similar to the PLA- $1 \mathrm{G}$ value, can be reduced to prevent adjacent-level ASD.

In our results, the ROM and von Mises stress at the fusion level were significantly lower in the PLA-4G model than that in the Bloc model. Moreover, these data showed that the PLA-4G model with anterior support was similar to the Tita model. Anterior support provided by a highstrength material, such as PLA-4G and Tita models, can prevent subsidence during the initial fusion period. This result is comparable to previous reports that showed that the cervical anterior plate system reduces the cage subsidence rate and enhances fusion rate after ACDF [2-4].

The von Mises stress at the adjacent segment level was lower in the PLA-1G model than in the Tita and PLA-4G models. Lower pressure to the adjacent disc was observed in the PLA-1G and Bloc model. In a study by Ji et al. [28], the incidence of ASD was higher after titanium plate-augmented ACDF than after cage-alone ACDF. The PLA-1G model is comparable to the second stage of the biodegradable system wherein the biodegradable plate is partially absorbed and weakened. Studies have reported that the plates maintain approximately $90 \%$ of their initial strength at 6 months after implantation, and the strength drops to $70 \%$ after approximately 9 months $[29,30]$. Consequently, pressure transmission to the adjacent level is reduced, reducing the ASD risk.

Our study has certain limitations. First, we assumed that the plate has full strength in the PLA-4G model in the early phase after surgery and weak strength after degradation. Although these data were from a preliminary study, the exact strength-versus-time graph of PLA remains unknown. Second, the biodegradable plate and screws lose strength when the plate and screw are undergoing degradation; furthermore, the degradation may not be continuous in nature. Rather than losing their strength in a linear pattern, the plate and screw may break abruptly during the postoperative period. Breakage of the plate and screw may increase the risk of injury to the esophagus or the surrounding tissues. Third, biodegradable plates can cause inflammation when they are biodegraded due to unknown biological effects or unknown mechanical effects of surrounding tissue. This reaction could cause some other complications. These situations were not simulated in the present study. 


\section{Conclusions}

The biodegradable cervical plate and screw system provides initial stiffness and enhances cervical fusion. When it gradually degrades, it may result in less stress on the adjacent segments in the long term. Further clinical studies are required to elucidate the efficacy and safety of biodegradable plates for ACDF.

\section{Conflict of Interest}

No potential conflict of interest relevant to this article was reported.

\section{Acknowledgments}

This work was supported by the Industrial R\&D program of MOTIE/KEIT (10052732).

\section{References}

1. Wang JC, McDonough PW, Endow KK, Delamarter RB. A comparison of fusion rates between singlelevel cervical corpectomy and two-level discectomy and fusion. J Spinal Disord 2001;14:222-5.

2. Joo YH, Lee JW, Kwon KY, Rhee JJ, Lee HK. Comparison of fusion with cage alone and plate instrumentation in two-level cervical degenerative disease. J Korean Neurosurg Soc 2010;48:342-6.

3. Pinder EM, Sharp DJ. Cage subsidence after anterior cervical discectomy and fusion using a cage alone or combined with anterior plate fixation. J Orthop Surg (Hong Kong) 2016;24:97-100.

4. Song KJ, Taghavi CE, Hsu MS, Lee KB, Kim GH, Song JH. Plate augmentation in anterior cervical discectomy and fusion with cage for degenerative cervical spinal disorders. Eur Spine J 2010;19:1677-83.

5. Veeravagu A, Cole T, Jiang B, Ratliff JK. Revision rates and complication incidence in single- and multilevel anterior cervical discectomy and fusion procedures: an administrative database study. Spine J 2014;14:1125-31.

6. Fountas KN, Kapsalaki EZ, Nikolakakos LG, et al. Anterior cervical discectomy and fusion associated complications. Spine (Phila Pa 1976) 2007;32:2310-7.

7. Cole T, Veeravagu A, Zhang M, Ratliff JK. Surgeon procedure volume and complication rates in an- terior cervical discectomy and fusions: analysis of a national longitudinal database. Clin Spine Surg 2017;30:E633-9.

8. Bazaz R, Lee MJ, Yoo JU. Incidence of dysphagia after anterior cervical spine surgery: a prospective study. Spine (Phila Pa 1976) 2002;27:2453-8.

9. Chin KR, Eiszner JR, Adams SB Jr. Role of plate thickness as a cause of dysphagia after anterior cervical fusion. Spine (Phila Pa 1976) 2007;32:2585-90.

10. Frempong-Boadu A, Houten JK, Osborn B, et al. Swallowing and speech dysfunction in patients undergoing anterior cervical discectomy and fusion: a prospective, objective preoperative and postoperative assessment. J Spinal Disord Tech 2002;15:362-8.

11. Martin RE, Neary MA, Diamant NE. Dysphagia following anterior cervical spine surgery. Dysphagia 1997;12:2-8.

12. Mechlin M, Thickman D, Kressel HY, Gefter W, Joseph P. Magnetic resonance imaging of postoperative patients with metallic implants. AJR Am J Roentgenol 1984;143:1281-4.

13. Chen M, Yang S, Yang C, et al. Outcomes observed during a 1-year clinical and radiographic follow-up of patients treated for 1- or 2-level cervical degenerative disease using a biodegradable anterior cervical plate. J Neurosurg Spine 2016;25:205-12.

14. Thomas KA, Toth JM, Crawford NR, et al. Bioresorbable polylactide interbody implants in an ovine anterior cervical discectomy and fusion model: threeyear results. Spine (Phila Pa 1976) 2008;33:734-42.

15. Panjabi MM, Crisco JJ, Vasavada A, et al. Mechanical properties of the human cervical spine as shown by three-dimensional load-displacement curves. Spine (Phila Pa 1976) 2001;26:2692-700.

16. Goel VK, Clausen JD. Prediction of load sharing among spinal components of a C5-C6 motion segment using the finite element approach. Spine (Phila Pa 1976) 1998;23:684-91.

17. Lee SH, Im YJ, Kim KT, Kim YH, Park WM, Kim K. Comparison of cervical spine biomechanics after fixed- and mobile-core artificial disc replacement: a finite element analysis. Spine (Phila Pa 1976) 2011;36:700-8.

18. Kubo S, Goel VK, Yang SJ, Tajima N. Biomechanical evaluation of cervical double-door laminoplasty using hydroxyapatite spacer. Spine (Phila Pa 1976) 2003;28:227-34. 
19. Pelker RR, Duranceau JS, Panjabi MM. Cervical spine stabilization: a three-dimensional, biomechanical evaluation of rotational stability, strength, and failure mechanisms. Spine (Phila Pa 1976) 1991;16:117-22.

20. Maurel N, Lavaste F, Skalli W. A three-dimensional parameterized finite element model of the lower cervical spine: study of the influence of the posterior articular facets. J Biomech 1997;30:921-31.

21. Moroney SP, Schultz AB, Miller JA. Analysis and measurement of neck loads. J Orthop Res 1988;6:71320.

22. Bydon M, Xu R, Macki M, et al. Adjacent segment disease after anterior cervical discectomy and fusion in a large series. Neurosurgery 2014;74:139-46.

23. Ahn SS, Paik HK, Chin DK, Kim SH, Kim DW, Ku MG. The fate of adjacent segments after anterior cervical discectomy and fusion: the influence of an anterior plate system. World Neurosurg 2016;89:42-50.

24. Elliott CA, Fox R, Ashforth R, Gourishankar S, Nataraj A. Magnetic resonance imaging artifact following anterior cervical discectomy and fusion with a trabecular metal cage. J Neurosurg Spine 2016;24:496501.

25. Jarvela T, Moisala AS, Sihvonen R, Jarvela S, Kannus $\mathrm{P}$, Jarvinen M. Double-bundle anterior cruciate ligament reconstruction using hamstring autografts and bioabsorbable interference screw fixation: prospective, randomized, clinical study with 2-year results. Am J Sports Med 2008;36:290-7.

26. Landes C, Ballon A, Ghanaati S, et al. Evaluation of the fatigue performance and degradability of resorbable PLDLLA-TMC osteofixations. Open Biomed Eng J 2013;7:133-46.

27. Kim WB, Hyun SJ, Choi H, Kim KJ, Jahng TA, Kim HJ. Long-term follow-up results of anterior cervical inter-body fusion with stand-alone cages. J Korean Neurosurg Soc 2016;59:385-91.

28. Ji GY, Oh CH, Shin DA, et al. Stand-alone cervical cages versus anterior cervical plates in 2-level cervical anterior interbody fusion patients: analysis of adjacent segment degeneration. J Spinal Disord Tech 2015;28:E433-8.

29. Claes LE, Ignatius AA, Rehm KE, Scholz C. New bioresorbable pin for the reduction of small bony fragments: design, mechanical properties and in vitro degradation. Biomaterials 1996;17:1621-6.

30. Peltoniemi HH, Tulamo RM, Pihlajamaki HK, et al. Consolidation of craniotomy lines after resorbable polylactide and titanium plating: a comparative experimental study in sheep. Plast Reconstr Surg 1998;101:123-33. 